\title{
Investigations on the sensing mechanisms in silicon nanowire Schottky-barrier field effect sensors
}

\author{
Sebastian Pregl ${ }^{1,2}$, Lotta Römhildt ${ }^{1}$, Walter M. Weber ${ }^{2}$, Larysa Baraban ${ }^{1}$, Jörg Opitz ${ }^{1,3}$, \\ Thomas Mikolajik ${ }^{2,4}$, and Gianaurelio Cuniberti, ${ }^{1,5}$ \\ ${ }^{1}$ Institute for Materials Science and Max Bergmann Center of Biomaterials, TU Dresden, 01062 \\ Dresden, Germany \\ research@nano.tu-dresden.de \\ ${ }^{2}$ NaMLab GmbH, 01187 Dresden, Germany \\ ${ }^{3}$ Fraunhofer Institute IZFP Dresden, 01109 Dresden, Germany \\ ${ }^{4}$ Institute for Semiconductors and Microsystems Technology, TU Dresden, 01187, Dresden, Germany \\ ${ }^{5}$ Division of IT Convergence Engineering, POSTECH, Pohang, Korea
}

\begin{abstract}
:
Surface functionalization of $\mathrm{NiSi}_{2}-\mathrm{Si}_{-} \mathrm{NiSi}_{2}$ nanowire heterostructures, acting as Schottky-junction field effect transistors (SB-FETs) represent a promising route for biosensor applications. Axially $\mathrm{Ni}$ silicidized silicon nanowires exhibit a very sharp metal-semiconductor interface, thus forming a well defined and reproducible Schottky barrier. These barriers determine the current through the wire and can be changed by small molecules chemiadsorbed on the nanowire surface. We report that surface modifications can alter the polarity of the devices. This severe influence on the charge transport implies ultrahigh sensitivity for nanowire SB-FETs.
\end{abstract}

Key words: Schottky-barrier, silicon nanowire, biosensor, field effect transistorSilicon

Nanowire based field effect transistors (FETs) have shown to be capable of label-free and real-time multiplexed detection of biochemical species [1]. The binding event of target molecules to a surface of the wires leads to a gating effect and therefore to a shift in threshold voltage. The reason for this is the high surface to volume ratio which makes the electronic properties of nanowires very sensitive to surface adsorbates and covalently bound molecules. For doped silicon nanowires with transparent contacts the pure field effect is claimed to be responsible for the sensing of charged molecules binding to the wire $[1,2]$. The field effect leads to an accumulation or depletion of charge carriers depending on wire doping type and charge of the target molecule. In this way the resistivity and current through the wire can be substantially changed.

Here we focus on an alternative system for sensors, namely undoped silicon nanowires in which the Schottky-junctions dominate the transport of charge carriers. In such systems novel mechanisms changing the contact resistance have to be conceived. It is assumed that changes of the transport through the Schottky barrier-FETs (SB-FETs) are governed by the interplay of many effects, i.e.
Schottky barrier height modulation, gate capacitance change and gating by surface charges like it was proposed for single walled carbon nanotubes $[3,4]$. Especially the field effect of charged molecules at the surface plays a crucial role for the sensing ability of the devices. It was shown that conduction and valence bands can be bent with electrical fields which leads to enhanced or reduced thermionic emission and tunneling through the energy barriers [5,6]. Our discussions presented in this paper are dedicated to the impact of biochemical functionalization of the nanowires on the charge transport, which is of high relevance in the field of biosensorics.

In the following, the growth procedure of the nanowires and device fabrication for our experiments is described. Undoped silicon nanowires are grown by catalytic chemical vapor deposition (CVD) [7]. Silane $\left(\mathrm{SiH}_{4}\right)$ is used as precursor and hydrogen as carrier gas with a composition of $\mathrm{SiH}_{4} / \mathrm{H}_{2}(10 / 1 \mathrm{p} / \mathrm{p})$ and a flow rate of $200 \mathrm{sccm}$. Growth is performed at a total pressure of $65 \mathrm{mbar}$ and a temperature of $450^{\circ} \mathrm{C}$. Gold nanoparticles with averaged diameter of aproximately $20 \mathrm{~nm}$, fabricated by BB International, are used as seed particles. Using this procedure, nanowires with a 
diameter of $20 \mathrm{~nm}$ and lengths up to $40 \mu \mathrm{m}$ are grown. Single nanowire devices are obtained by drying droplets of isopropanol containing suspended nanowires on chips with recessed cobalt electrodes. Electrical connection is provided by electroless nickel deposition, which covers cobalt electrodes selectively, as described elsewhere [8]. For silicon nanowires contacted to nickel electrodes, annealing leads to axial silicidation which can form an atomically flat metal - semiconductor interface [9].

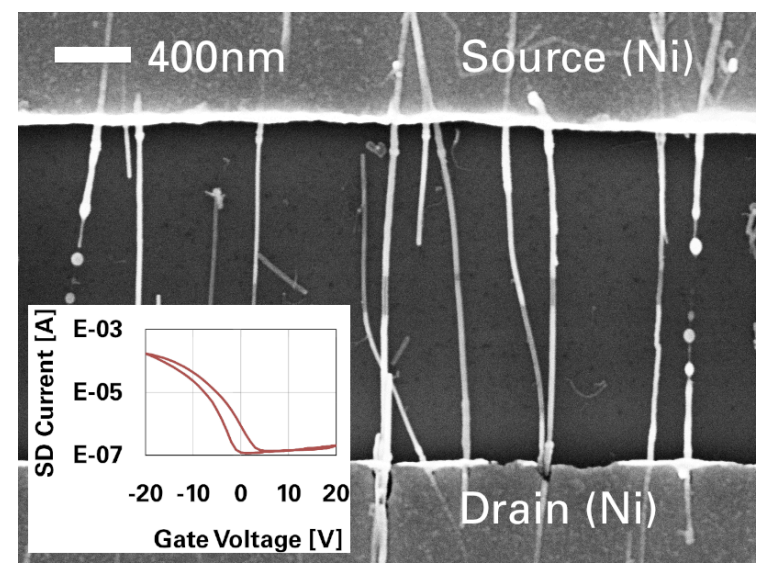

Fig. 1: SEM top view image detail of a parallel array of SB-FETs. Brighter parts of the nanowires are NiSi phases, formed after alloying. Fully silicidized (metallic) wires were electrically burned before measurements. The silicon nanowires are printed on a $\mathrm{SiO}_{2}$ / Si back gate stack. The inset shows the transfer characteristics of the device.

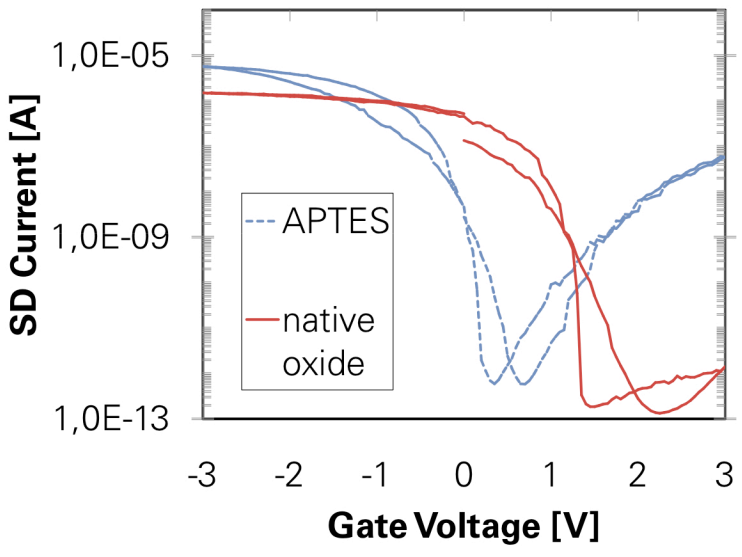

Fig. 2: Transfer characteristics of a back gated single nanowire SB-FET. Back gate thermal Sioxide thickness is $20 \mathrm{~nm}$, source drain voltage was $0.5 \mathrm{~V}$. Different colors represent different stages of surface modifications of the same nanowire. Starting with the native oxide shell (red, solid) of the wire a functionalization was done to obtain amino terminated wires (blue, dotted).
The current through the resulting Schottky $\mathrm{NiSi}_{2}$ / Si junctions can be efficiently manipulated by external electric fields. This can be seen from the high on/off current ratio of the transfer characteristics obtained in back gate configuration of $10^{6}$ [Figure 2]. Although the on-current densities are high, a drawback is their small total current of single wire devices $\left(\mathrm{I}_{\mathrm{on}} @ 0.5 \mathrm{~V}_{\mathrm{sd}} \sim 1 \mu \mathrm{A}\right)$ compared to large planar and thin film devices.

To obtain higher currents and thus higher signal, parallel arrays of nanowire SB-FETs are fabricated. The contact printing technique is used to transfer nanowires from the growth substrate to the chip substrate with high density and pre-defined orientation [10]. The arrays of silicon nanowires are contacted in parallel by interdigital electrode structures fabricated using UV-lithography. A significant enhancement of on-currents could be reached with these parallel arrays. A section of a parallel array device with interdigitated electrodes and an area of $0.06 \mathrm{~mm}^{2}$ is depicted in [Figure 1]. The inset shows the transfer characteristics of the device. The device shows on currents of $171 \mu \mathrm{A}$ at a drainsource voltage of $0.5 \mathrm{~V}$. The characteristic shape of single nanowire devices is maintained. Such high-current-FET systems are appropriate for sensor systems with high readout currents and large active sensor area. Importantly, statistics on our results strongly imply that averaging the signal of multiple nanowire SB-FETs by assembling them in parallel makes the device and electrical characteristics more reliable and stable.

Surface modifications performed on single nanowires have shown a non trivial change of the transfer characteristics which cannot be explained by surface charge and gating effects alone. Devices were functionalized using (3Aminopropyl)-triethoxysilane (APTES; SigmaAldrich $\mathrm{GmbH}$ ). Prior to functionalization the surfaces are cleaned with absolute ethanol. The surface activation of the devices with nanowires exhibiting a native oxide shell is performed using air plasma for $2 \mathrm{~min}$. The devices are immersed in a 2 vol\% APTES solution in an ethanol $/ \mathrm{H}_{2} \mathrm{O}$ mixture (95/5 vol\%) for $1 \mathrm{~h}$ after hydrolysis for $10 \mathrm{~min}$ [11]. An additional bake for $10 \mathrm{~min}$ at $110^{\circ} \mathrm{C}$ was performed to assure the crosslinking of the attached silane molecules. The functionalized devices are stored in $\mathrm{N}_{2}$ atmosphere until being measured. The change of the transfer characteristics of the devices is depicted in [Figure 2]. Schottky-barrier theory predicts ambipolar IV-characteristics for undoped Si- 
nanowire SB-FETs with $\mathrm{Ni}$ contacts [5]. Nevertheless, unfunctionalized devices with a native oxide shell exhibit unipolar p-type behaviour. Although this could be attributed to unintentional doping, the low off-currents rule out this explanation [12]. It is known that thermal oxidation of the nanowires transforms the device characteristics to ambipolar [5]. In our experiments, a surface functionalization with APTES led to a similar effect although the underlying reasons apparently have different origin. Off- currents were changed by 4 orders of magnitude which illustrates the high impact of surface effects.

Conclusively, we discussed the influence of the surface functionalization on the characteristics of silicon nanowire SB-FETs. The polarity of the FETs could be changed from unipolar ( $p$-type) to ambipolar via surface functionalization of the native oxide shell of the nanowires with APTES. The strong change of currents in the electron dominated branch indicate a very high sensitivity for surface interactions which promises nanowire SBFETs to become sensor platforms for analytes of lowest concentrations. Sensor elements with a high active area and sufficient high output current can be produced with parallel arrays of nanowire SB-FETs. Our experiments are supported by theoretical modeling of the SBFET device and the electrostatic surounding [13].

\section{Acknowledgements}

This work was supported by the European Union (European Social Fund) and the Free State of Saxony (Sächsische Aufbaubank) in the young researcher group 'InnovaSens' (SAB-Nr. 080942409). G.C. further acknowledges the World Class University program sponsored by the South Korean Ministry of Education, Science, and Technology Program, Project No. R31-2008-
000-10100-0. We thank A. Jahn (TU Dresden) for helpful discussions and his assistance with UV-lithography. We thank I. Mönch (IFW Dresden) for the fabrication of masks for UVlithography.

\section{References}

[1] G. Zheng, C. Lieber, Nature Biotechnology 23, 1294 - 1301 (2005); doi:10.1038/nbt1138

[2] X. P. A. Gao, C. M. Lieber, Nano Lett. 10, 547552 (2010); doi: 10.1021/n19034219

[3] I.Heller, C. Dekker, Nano Letters 8, 591-595 (2008); doi: 10.1021/nI072996i

[4] R. J. Chen, H. Dai, J. AM. CHEM. SOC. 126, 1563-1568 (2004); doi: 10.1021/ja038702m

[5] A. Heinzig et al. Nano Lett. (2011); doi: 10. $1021 / \mathrm{nl} 203094 \mathrm{~h}$

[6] D. Martin et al. Physical Review Letters 107, 216807 (2011); doi: 10.1103/PhysRevLett. 107.216807

[7] Y. Cui, C. Lieber, J. Phys. Chem. B 104, 52135216 (2000); doi: 10.1021/jp0009305

[8] M. Liebau, E. Unger, G. S. Duesberg, A. P. Graham, R. Seidel, F. Kreupl, and W. Hoenlein, Appl. Phys. A 77, 731-734 (2003); doi: 10.1007/s00339-003-2207-4

[9] K. Ogata et al, 2011 Nanotechnology 22 365305; doi:10.1088/0957-4484/22/36/365305

[10] Z.Fan, Nano Letters 8, 20-25 (2008); doi: $10.1021 / \mathrm{nl} 071626$

[11] V. Dugas, Y. Chevalier, In: Zourob, M., editor. , Recognition Receptors in Biosensors. 1st ed. Berlin: Springer, Part 1, 47-134 (2010); doi: 10.1007/978-1-4419-0919-0 2

[12] W. M. Weber, F.Kreupl, phys. stat. sol. (b), 16 (2006) ; doi: 10.1002/pssb.200669138

[13] D. Nozaki, J. Kunstmann et al., Nanotechnology 22, 325703 (2011); doi: $10.1088 / 0957-4484 / 22 / 32 / 325703$ 